Musées, Patrimoine et Culture scientifiques et techniques

$108 \mid 2006$

novembre-décembre 2006

\title{
De la reproduction du ciel à la simulation des connaissances humaines sur l'Univers
}

\section{Marc Girard et Nelly Dumas}

\section{(2) OpenEdition \\ Journals}

Édition électronique

URL : http://journals.openedition.org/ocim/1321

DOI : 10.4000/ocim.1321

ISSN : 2108-646X

Éditeur

OCIM

Édition imprimée

Date de publication : 25 novembre 2006

ISSN : 0994-1908

Référence électronique

Marc Girard et Nelly Dumas, « De la reproduction du ciel à la simulation des connaissances humaines sur l'Univers », La Lettre de I'OCIM [En ligne], 108 | 2006, mis en ligne le 12 mars 2014, consulté le 01 mai 2019. URL : http://journals.openedition.org/ocim/1321 ; DOI : 10.4000/ocim.1321 


\section{De la reproduction du ciel à la simulation des connaissances humaines sur l'Univers}

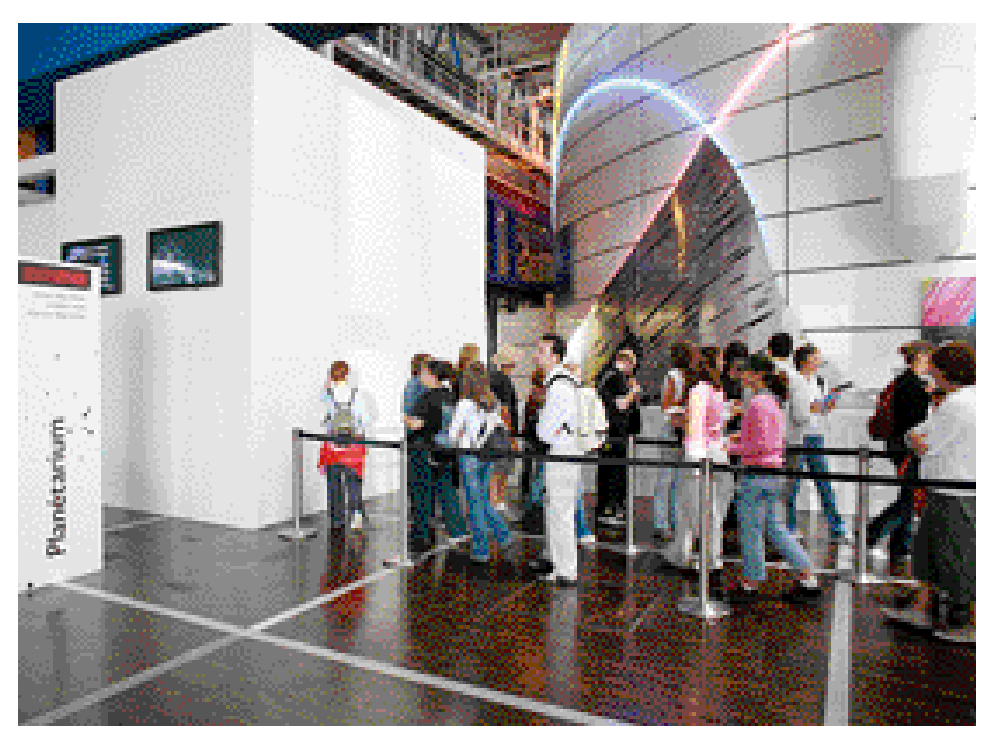

L'entrée du nouveau planétarium de la Cité des Sciences et de l'Industrie ○) Cité des Sciences et de l'Industrie

* Marc Girard est directeur des Expositions à la Cité des Sciences et de l'Industrie m.girard@cite-sciences.fr Nelly Dumas est chef du département Planétarium à la Cité des Sciences et de l'Industrie n.dumas@cite-sciences.fr

\section{Marc Girard et Nelly Dumas *}

Confrontée au vieillissement technique de son planétarium, la Cité des Sciences et de I'Industrie a récemment modernisé son équipement : les auteurs analysent l'ensemble des raisons qui ont présidées à cette évolution et présentent les nouvelles technologies choisies, tout en mettant l'accent sur la nécessaire coopération des membres du réseau des planétariums, notamment pour tout ce qui concerne l'échange et la coproduction des spectacles astronomiques.

Pourquoi rénover le planétarium de la Cité des Sciences et de l'Industrie ?

Lors de sa création, au milieu des années 1980, la Cité des Sciences et de l'Industrie s'était dotée d'un planétarium dont la taille - 23 mètres de diamètre, 300 places - et la situation - au cœur des espace d'exposition - témoignaient du rôle primordial qui lui était conféré parmi les médias d'accès à la culture scientifique que proposait le nouvel établissement. Complexité de la production de spectacles, maintenance coûteuse : les équipements techniques sophistiqués installés à l'époque autour d'un simulateur d'étoiles optique ont vieilli et les choix technologiques d'alors ont été remis en cause par les mutations technologiques de l'ère du tout numérique.

De plus, certains de nos visiteurs, amateurs des effets spéciaux des grands films « cosmiques », des jeux vidéo « inter galactiques », habitués des salles immersives ou hémisphériques, sont devenus plus exigeants en matière de spectacles astronomiques. 
D'autres, bien qu'attachés à la dimension éducative et culturelle de nos programmes, souhaitent que, sans rien renier de nos exigences, nous nous efforcions de moderniser le " spectacle de planétarium de papa ». C'est donc confronté à l'obsolescence technique de notre planétarium et aux nouvelles attentes de ses spectateurs que nous en avons entrepris la rénovation avec pour guide une typologie des spectacles que nous souhaitions proposer et le souci de tirer le meilleur parti des possibilités offertes par les techniques numériques.

\section{Quelle programmation pour quels publics?}

Le public du planétarium, comme celui de la Cité des Sciences elle-même, n'est pas homogène. Nous accueillons principalement deux grandes catégories de public : les groupes scolaires - environ $40 \%$ de notre fréquentation - et les visiteurs individuels venus majoritairement en famille - environ $60 \%$ de notre fréquentation.

Ces deux catégories de public ont des attentes distinctes :

- les accompagnateurs de groupes scolaires souhaitent, pour la plupart, des séances pédagogiques, conduites par un médiateur scientifique et à fort contenu didactique; ils ne réclament pas que soit donné un caractère particulièrement divertissant ou spectaculaire à une initiation à l'Astronomie et souhaitent des spectacles ayant un lien avec les programmes scolaires. C'est pour ce public que nous travaillons à des conférences-animations sur la mécanique céleste, la structure solaire ou encore la spectroscopie ;

- la demande du public individuel ou familial est assez différente. Beaucoup de nos visiteurs ont déjà à l'esprit le Futuroscope, la Géode et d'autres salles immersives. Ils souhaitent manifestement des spectacles multimédias, alliant la simulation du ciel étoilé à des images animées et à une bande son de qualité. En clair, ce public en quête de loisirs culturels, cherche un divertissement instructif et se révèle sensible à l'aspect spectaculaire des productions qui lui sont proposées. Ce public est aussi celui auquel nous proposons de sortir des limites de l'Astronomie pure, pour présenter également des spectacles sur la planétologie, le climat ou encore le volcanisme.

Concernant les amateurs d'Astronomie déjà initiés qui viennent au planétarium, nous projetons de les inviter à des soirées spéciales - au moment des solstices et des équinoxes par exemple - pour leur présenter des conférences-animations portant sur les événements astronomiques du trimestre à venir.

\section{Des choix techniques adaptés}

Depuis quelques années, les nouvelles technologies numériques révolutionnent le monde des planétariums. Ces technologies permettent de ne plus se cantonner à la reproduction d'un ciel tel qu'on peut le voir depuis la Terre, mais autorisent de grands voyages dans l'Espace et le Temps, montrant avec la même apparente facilité le ciel vu depuis la Terre il y a des milliers d'années, ou le ballet des satellites de Jupiter autour de leur planète en images de haute résolution.

On peut se risquer à dire qu'en choisissant ces nouvelles techniques, on passe de la reproduction du ciel et de ses étoiles à la simulation de ce que nous savons de l'Univers.

Le planétarium de la Cité des Sciences et de I'Industrie

- un dôme de 21,50 mètres de diamètre - une inclinaison du dôme à $3,5^{\circ}$, parallèle à la pente des gradins

- 272 places orientées dont 7 réservées aux personnes handicapées

- un simulateur In Space System de RSA Cosmos - 8 vidéoprojecteurs Barco DLP SIM5+

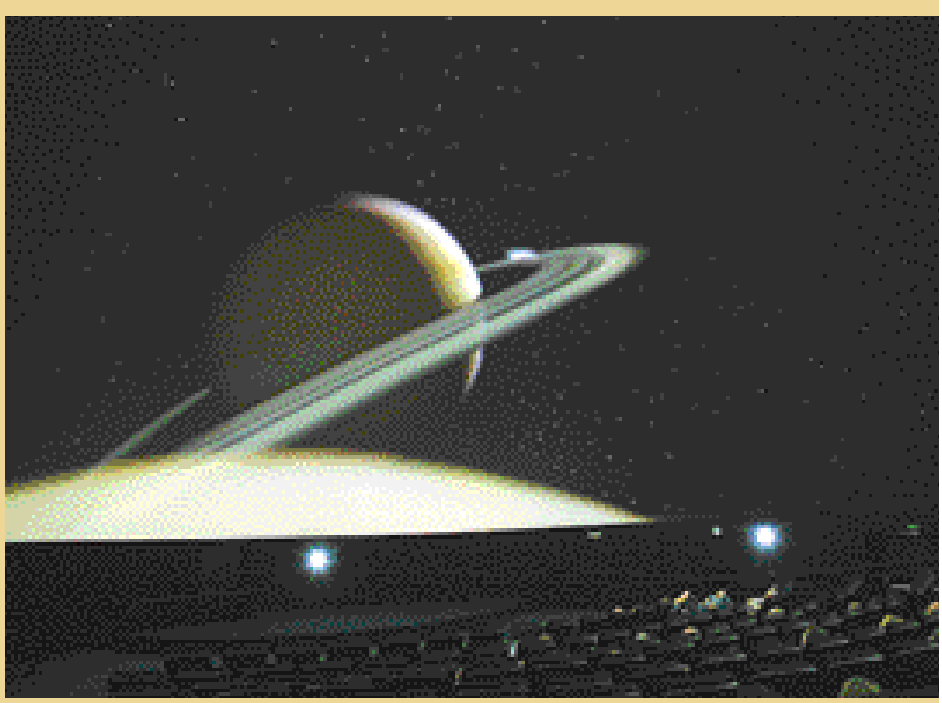

Dans la salle de projection, le dôme de 21,50 mètres de diamètre surplombe les 272 places. (๑) Cité des Sciences et de l'Industrie 


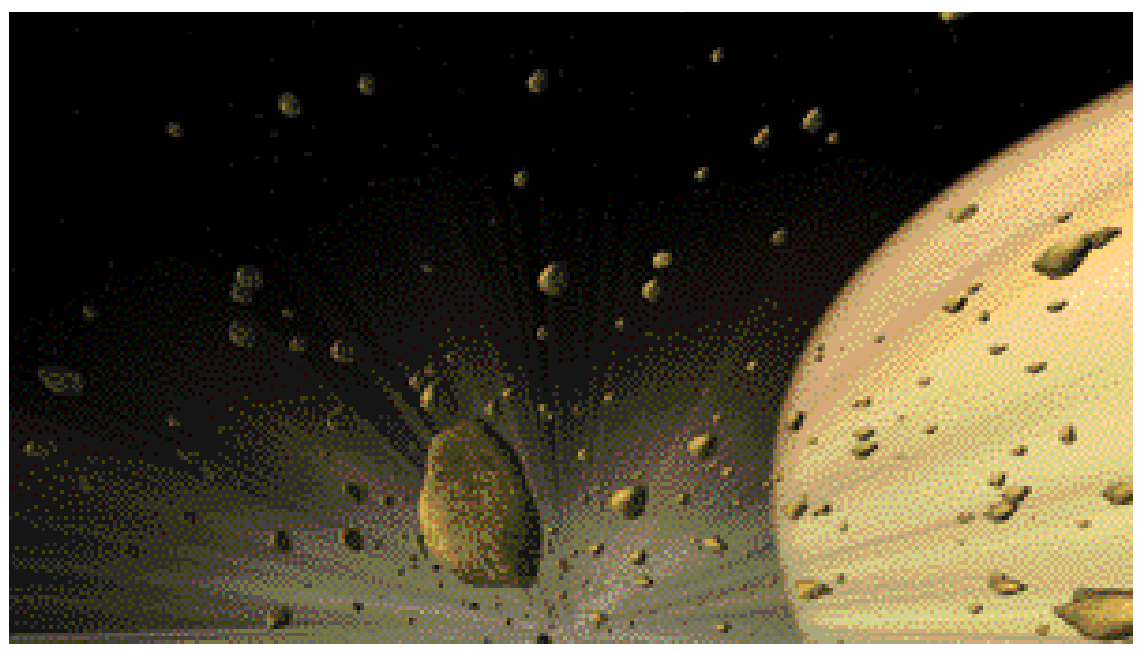

Saturne, une image du spectacle Oasis dans l'espace produit par la société Spitz. ๑) Cité des Sciences et de l'Industrie

Après une période attentiste - 1998-2002 - au cours de laquelle la technologie s'est consolidée, de plus en plus de salles ont franchi le pas.

La technologie choisie, à savoir un système vidéo pleine voûte, a maintenant atteint un niveau de résolution qui permet de diffuser simultanément le ciel, en temps réel, issu d'une base de données scientifique, et des images, fixes ou animées, à partir d'une source numérique enregistrée. Ces systèmes comportent, de plus, leur propre automation, capable de gérer les autres composantes des spectacles : son, lumière... En résumé, et en simplifiant, 8 vidéoprojecteurs diffusant les images issues d'ordinateurs remplacent tous les composants de l'ancien système technique comptant 110 projecteurs de diapositives, un simulateur astronomique complexe et 3 vidéoprojecteurs... Nous attendons évidemment de cette modernisation technique des économies en matière de maintenance. Mais surtout, nous comptons sur le potentiel pédagogique considérable du système vidéo pleine voûte : ciel en 3D, système solaire et objets célestes texturés, souplesse d'utilisation des différents cercles de coordonnées, contours de constellations, paramètres environnementaux - jour, nuit atmosphère, nuages...

La numérisation qui permet d'intégrer à une création des images d'origines diverses, offre aussi la possibilité de réagir beaucoup plus rapidement à l'actualité : en intégrant des images fixes ou animées pour réactualiser des spectacles, en créant des modules courts, associant images et simulateur pour, par exemple, situer les dernières planètes extra solaires ou pour présenter le déplacement sur la voûte céleste d'une comète récemment découverte.

Une autre caractéristique très riche des possibilités du système vidéo pleine voûte est la facilité d'adaptation d'un spectacle d'une salle à une autre, même avec des configurations de projecteurs différentes, le découpage du dôme master du spectacle en différents canaux étant effectué par chaque planétarium en fonction de sa configuration.

Entre planétariums numériques, la circulation des spectacles devrait sintensifier. Une sorte de marché de modules animés, fragments dont le montage constitue des spectacles ou enrichit des animations, pourrait également se développer. Cette capacité a beaucoup d'importance à nos yeux. Il nous parait en effet indispensable, pour bénéficier d'images et de spectacles à des coûts raisonnables, de pouvoir les échanger entre planétariums et en organiser la coproduction avec eux.

L'ambition de faire une large place à l'échange et à la coproduction dans les modes de production du planétarium de la Cité des Sciences et de l'Industrie nous a conduit à tout mettre en œuvre pour faciliter ce que l'on pourrait qualifier " l'interopérabilité » de nos productions et de nos équipements avec ceux du réseau des planétariums. L'orientation de notre salle, après des années d'exploitation avec un plan de sièges concentriques autour du simulateur d'étoiles installé en position centrale, s'est imposée pour nous permettre d'être plus facilement en mesure d'accueillir les productions des planétariums les plus récents qui, tous, ont choisi de se doter de salles orientées.

La configuration de la salle a donc été modifiée. Les sièges ne sont plus concentriques, mais orientés dans la même direction de façon à ce que chaque spectateur puisse voir la même chose que son voisin. Le nombre de places est désormais de 265, plus 7 places réservées aux personnes handicapées. Cette décision a été prise à l'issue d'un large débat interne : fallait-il renoncer au " réalisme » d'une configuration qui fait converger le regard des spectateurs vers le zénith du dôme écran au profit d'une configuration facilitant l'expression du conférencier-animateur qui peut faire face à l'ensemble de son auditoire ? Fallaitil maintenir un plan de salle favorable à l'observation 
d'une réplique de la voûte céleste ou privilégier l'orientation de la salle qui facilite l'écriture de spectacles astronomiques captivants et dynamiques ?

Il nous est apparu que, outre son caractère favorable à « l'interopérabilité », l'orientation de la salle était la plus adaptée à la mixité de ses usages : conférences-animations pour nos spectateurs les plus sensibles à l'apport pédagogique de leur visite, spectacles astronomiques pour les spectateurs à la recherche d'un moment de loisir de qualité.

Le choix des vidéoprojecteurs chargés de la diffusion des images a, lui aussi, été longuement débattu en fonction de la programmation que nous nous proposions d'offrir à nos visiteurs et de la taille importante de notre dôme. Ainsi, après de soigneux essais comparatifs, avons-nous choisi 8 projecteurs DLP plutôt que des projecteurs CRT. Ceux-ci, qui, il y a peu encore, constituaient l'équipement standard des planétariums en raison de la qualité du noir de fond de ciel qu'ils délivrent, pâtissent de coûts de maintenance très élevés. Mais ce ne fut pas pour nous l'argument décisif. Décidés à programmer aussi bien des conférences-animations exigeant un ciel « réaliste » que des spectacles astronomiques exigeant un rendu de sources vidéo de qualité, nous avons opté pour des vidéoprojecteurs de technologie DLP. Certes, le fond de ciel est moins noir, mais la profondeur de champ, la définition et la brillance des étoiles, sans parler de l'extrême qualité de la diffusion de vidéos ont emporté notre décision.

\section{Le programme inaugural}

Le planétarium propose, chaque jour, de 5 à 7 séances en fonction de l'affluence observée à différentes périodes de l'année. Nous nous efforçons également que les expositions à l'affiche à la Cité des Sciences trouvent un écho dans les programmes du planétarium, ne serait-ce que parce que celles-ci environnent notre salle.

Deux conférences-animations sont proposées chaque jour en début de journée et en début d'après-midi pour tenir compte des contraintes particulières des groupes scolaires. À ciel owvert : ce titre générique recouvre des séances d'une durée d'une vingtaine de minutes, programmées à l'aide du simulateur et animées en direct par des médiateurs scientifiques de l'établissement. Les thèmes varieront et seront choisis parmi des sujets assez classiques dans les planétariums : Le tour du système solaire, Le ciel du mois, Voyage dans les constellations, Étoiles et mythologies, Vie et mort des étoiles, Ombres et lumières, La couleur du ciel... Les trois à cinq autres séances, seront consacrées à la diffusion de spectacles astronomiques spectaculaires que nous louons avant
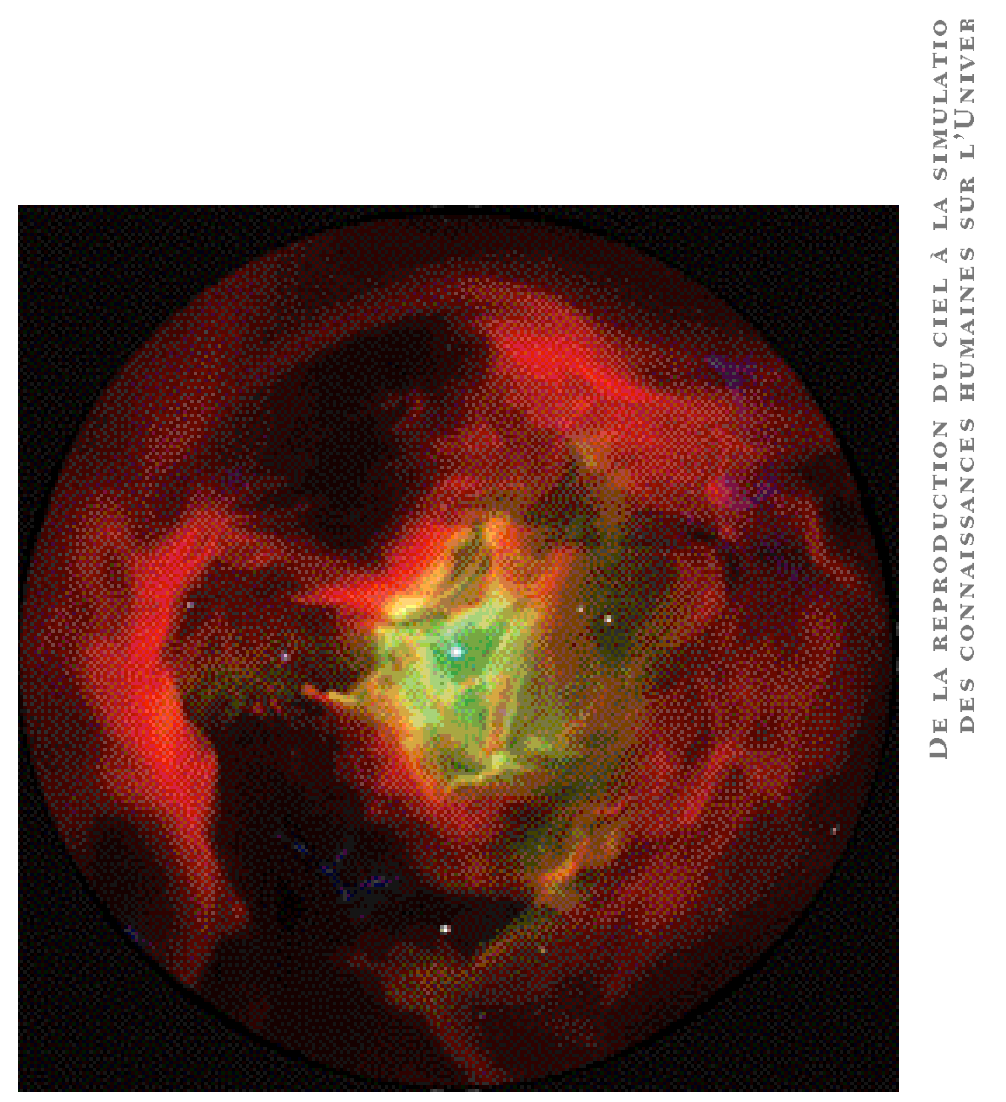

Le spectacle Sommes-nous seuls dans I'Univers est une production du Hayden planétarium de New York. (c) Cité des Sciences et de l'Industrie

d'être en mesure d'en produire - ou d'en coproduire nous-mêmes. Nous avons choisi pour la réouverture du planétarium Oasis dans l'Espace, un spectacle de 23 minutes produit par la société Spitz, écho à l'exposition L'Eau pour tous; nous programmons depuis l'automne La recherche de la Vie: sommes-nous seuls dans l'Univers ?, produit par le Hayden planétarium de New York, simultanément à l'ouverture d'une exposition consacrée au même sujet.

\section{Un objectif de production}

La Cité des Sciences et de l'Industrie a consenti à intégrer à son plan de rénovation le lourd investissement que constitue la modernisation de son planétarium pour en augmenter la fréquentation. Cet objectif sera atteint à court terme grâce à l'enrichissement de la programmation que permet ce nouveau système technique. Mais l'enjeu pour les années à venir est de mettre ce splendide outil technique au service du développement d'une offre originale de spectacles et d'animations, exprimant des contenus scientifiques incontestables et témoignant d'une forte créativité audiovisuelle. Ces productions devront associer astronomes et astrophysiciens, médiateurs scientifiques, réalisateurs et infographistes autour de projets associant vraisemblablement plusieurs planétariums. C'est en tout cas la voie sur laquelle nous souhaitons nous engager. 